\title{
Insulator to Metal Transition in Fluid Hydrogen
}

Randolph Q. Hood and Giulia Galli

\section{January 15, 2003}

U.S. Department of Energy

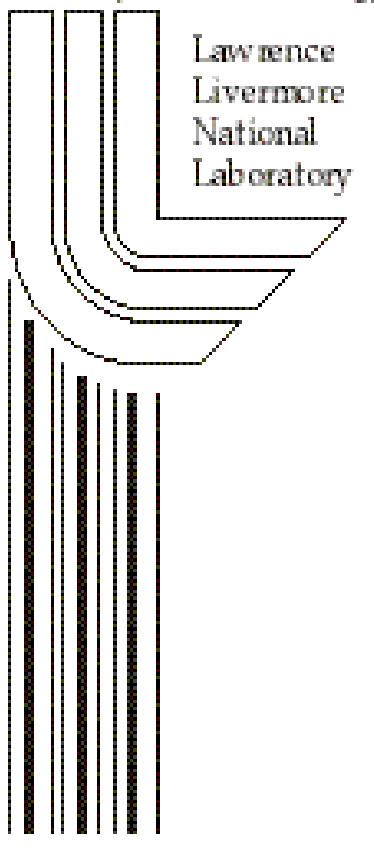




\section{DISCLAIMER}

This document was prepared as an account of work sponsored by an agency of the United States Government. Neither the United States Government nor the University of California nor any of their employees, makes any warranty, express or implied, or assumes any legal liability or responsibility for the accuracy, completeness, or usefulness of any information, apparatus, product, or process disclosed, or represents that its use would not infringe privately owned rights. Reference herein to any specific commercial product, process, or service by trade name, trademark, manufacturer, or otherwise, does not necessarily constitute or imply its endorsement, recommendation, or favoring by the United States Government or the University of California. The views and opinions of authors expressed herein do not necessarily state or reflect those of the United States Government or the University of California, and shall not be used for advertising or product endorsement purposes.

This work was performed under the auspices of the U. S. Department of Energy by the University of California, Lawrence Livermore National Laboratory under Contract No. W-7405-Eng-48.

This report has been reproduced directly from the best available copy.

Available electronically at http://www.doc.gov/bridge

Available for a processing fee to U.S. Department of Energy

And its contractors in paper from

U.S. Department of Energy

Office of Scientific and Technical Information

P.O. Box 62

Oak Ridge, TN 37831-0062

Telephone: (865) 576-8401

Facsimile: (865) 576-5728

E-mail: reports@adonis.osti.gov

Available for the sale to the public from

U.S. Department of Commerce

National Technical Information Service

5285 Port Royal Road

Springfield, VA 22161

Telephone: (800) 553-6847

Facsimile: (703) 605-6900

E-mail: orders@ntis.fedworld.gov

Online ordering: http:/ / www.ntis.gov/ordering.htm

\section{OR}

Lawrence Livermore National Laboratory

Technical Information Department's Digital Library

http:/ / www.llnl.gov/tid/Library.html 


\title{
Insulator to metal transition in fluid hydrogen
}

\author{
Randolph Q. Hood and Giulia Galli \\ Lawrence Livermore National Laboratory, Livermore, CA 94550
}

\begin{abstract}
We have investigated the insulator to metal transition (ITM) in fluid hydrogen using first principles simulations. Both density functional and quantum Monte Carlo calculations show that the electronic energy gap of the liquid vanishes at about 9 fold compression and $3000 \mathrm{~K}$. At these conditions the computed conductivity values are characteristic of a poor metal. These findings are consistent with those of recent shock wave experiments but the computed conductivity is larger than the measured value. From our ab-initio results we conclude that the ITM is driven by molecular dissociation rather than disorder and that both temperature and pressure play a key role in determining structural changes in the fluid.
\end{abstract}

The search for metallic hydrogen has been the subject of investigation for almost a century[1,2]. Solid hydrogen has been shown[3] to become opaque at $320 \mathrm{GPa}$ but its metallization has not been observed experimentally and it is predicted at $\simeq 450 \mathrm{GPa}$. Liquid hydrogen has instead been found[4] to become a poor metal at the relatively low pressure of $\simeq 140 \mathrm{GPa}$ and $\simeq 3000 \mathrm{~K}$.

Based on empirical free-energy models, it was suggested that in the fluid metallization occurs in a molecular phase, by a mechanism similar to band gap closing in a disordered semiconductor[4]. This picture is not supported by tight-binding (TB)[5] and first principles molecular dynamics (FPMD) simulations[6] carried out at pressure $(\mathrm{P})$ and temperature $(\mathrm{T})$ conditions corresponding to the experimental insulator to metal transition (ITM) region. These calculations found that in the metallic fluid a significant proportion of molecules are dissociated, and they pointed at a correlation between molecular dissociation and finite DC conductivity. In general first principles calculations of compressed hydrogen $[7,8]$ have shown that the fluid is a complex, condensed state which cannot be simply described as a mixture of constituents such as atoms, molecules and ions, as it is assumed in chemical models $[4,9,10]$.

However the accuracy and validity of both TB and FPMD simulations near the ITM have also been questioned[11] for several reasons. TB simulations rely on semi-empirical parameters and they have limited predictive capabilities. FPMD is an ab-intio, predictive method, which however relies on the local density approximation (LDA) to density functional theory (DFT). Although no systematic study is yet available on the accuracy of LDA in describing the electronic properties of disordered systems, LDA in known to underestimate electronic gaps of crystalline solids and molecules. This error affects computed conductivities and might affect as well predicted metallization densities.

In this paper we present a series of first principles molecular dynamic simulations and quantum Monte Carlo calculations (QMC) aimed at determining the compression ratio and pressure at which the ITM transition takes place in fluid hydrogen. Determining accurate met- allization densities is key to the understanding of the hydrogen phase diagram, since in shock wave experiments pressure and conductivities are measured, while densities and temperature are estimated based on empirical models[4]. We also present a detailed study of the structural properties of the fluid and show that the ITM is driven by molecular dissociation, with temperature as well as pressure playing a key role in determining structural changes upon compression. To our knowledge, first principles theories have never been applied to determine the metallization density and pressure of fluid hydrogen. In addition, our QMC results for liquid hydrogen represent the first calculations of the electronic properties of the fluid beyond DFT, as well as the first QMC calculations of the electronic properties of a condensed, disordered system.

In our FPMD simulations[12] we used a generalized gradient approximation (GGA) to the exchange and correlation energy[13] and FPMD cells containing up to 992 atoms. In particular, we carried out constant volume simulations with cells containing 124 deuterium atoms at densities of $0.67,1.0,1.5$ and $2.35 \mathrm{~g} / \mathrm{cm}^{3}$ corresponding to 4-, 6-, 9- and 14-fold compression, respectively. At 9-fold compression we repeated our simulations using a 992 atom cell. Electronic gaps at different densities for a series of FPMD snapshots were computed using fixednode diffusion quantum Monte Carlo (DMC).

We first describe the evolution of the atomic structure as the fluid is brought from 4- to 14-fold compression; we will then analyze the corresponding changes of the electronic structure and discuss in detail calculations of the conductivity in the ITM region. The calculated deuteron-deuteron $\left(\mathrm{D}_{2}\right)$ pair correlation functions $[g(r)]$ at 4-, 6-, 9-, and 14-fold compression and $3000 \mathrm{~K}$ (not shown) exhibit a first maximum, the intramolecular peak, moving outward and decreasing in magnitude with increasing density. Correspondingly, the first minimum increases in magnitude, indicating that the liquid is becoming less molecular upon compression. At 14fold compression the $g(r)$ is almost structure-less. In our simulations at $3000 \mathrm{~K}$ and compressions larger than four, we find that $\mathrm{D}_{2}$ pairs are forming and breaking on time 
scales $\left(10^{-14}\right.$ sec.) of the order of the vibrational period of an isolated $\mathrm{D}_{2}$ molecule. At 9-fold compression intraand inter-molecular distances overlap. Therefore if we use geometrical proximity of two deuterons as a criterion to define a molecule, the very concept of molecule becomes ill defined. In order to understand the bonding nature of $\mathrm{D}_{2}$ pairs we used maximally localized Wannier functions (MLWF)[14] to locate centers of charge in the system and to investigate whether two deuterons which are geometrically close to one another are also bound. At 4-fold compression and $3000 \mathrm{~K}$ the centroid of each MLWF is located near the midpoint of two neighboring deuterons. As the liquid is compressed, the MLWF cen-

TABLE I: Calculated percent dissociation in liquid deuterium for simulation cells with 124 atoms at the compression ratio $\rho / \rho_{0}$, temperature $\mathrm{T}$, and pressure $\mathrm{P}$ using the geometrical and Wannier definitions (see text). Statistical errors on $\mathrm{P}$ are less than $5 \%$

\begin{tabular}{crccc}
$\rho / \rho_{0}$ & $\mathrm{~T}(\mathrm{~K})$ & $\mathrm{P}(\mathrm{GPa})$ & \multicolumn{2}{c}{ geometrical } \\
\hline 4 & 3000 & 12 & 9 & 0 \\
4 & 10000 & 35 & 45 & 35 \\
6 & 3000 & 34 & 22 & 21 \\
6 & 10000 & 79 & 46 & 42 \\
9 & 3000 & 110 & 41 & 38 \\
14 & 3000 & 350 & 50 & 50 \\
\hline
\end{tabular}

troids move away from the midpoint of the geometrically defined bond (of length $d$ ), indicating the formation of pairing states rather different from those present at low pressure. We have used two different definitions of paired states, or "molecules". The first is based solely on a geometrical criterion[6] and involves all $\mathrm{D}_{2}$ pairs that are separated by less than $r_{c u t}$, the value at which $g(r)$ integrates to one. Of the $\mathrm{D}_{2}$ comprising these pairs, a deuteron with only one neighbor within $r_{c u t}$ is said to belong to a "molecule". The second definition incorporates the positions of the Wannier centers, and considers all $\mathrm{D}_{2}$ separated by less than $r_{c u t}$ that in addition have a Wannier center located within $d / 2$. Table I shows the percent dissociation using the two definitions at different $\rho$ and T. The geometrical definition tends to give larger dissociation fractions. However, at the conditions where the experiments observe a poor metal - 9-fold compression and $3000 \mathrm{~K}$ - both definitions give very similar dissociation fractions ( $41 \%$ and $38 \%$ ). These results are consistent with those of previous FPMD simulations[6] that found a geometrical dissociation fraction of $42 \%$ at these conditions, but they are very different from predictions given by simple models[4] of around $5 \%$. If the liquid is further compressed from 9- to 14-fold compression on the $3000 \mathrm{~K}$ isotherm, the number of dissociated molecules slightly increases to about $50 \%$. With increasing density and temperature the so-called "molecules" typically

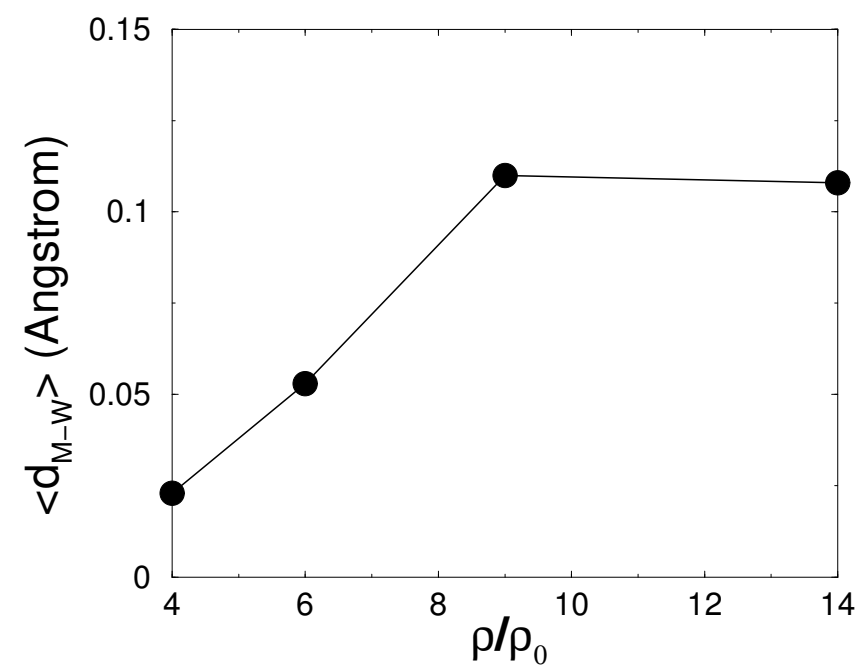

FIG. 1: The configurational averaged separation of the Wannier center from the midpoint of the two deuterons which comprise a "molecule" (Wannier definition) versus the compression ratio of the density for simulations with 124 atoms at $3000 \mathrm{~K}$. The line is a guide to the eye.

become more distorted and the average spread of MLWFs increases. Fig. 1 shows how the average position of a Wannier center deviates from the midpoint of the two deuterons in each "molecule". This molecular distortion is proportional to the magnitude of the electronic polarization of each "molecule"[15] and its behavior as a function of pressure indicate a bonding transformation occurring in the fluid at about 9-fold compression.

We notice that bonding transformations analogous to those induced by pressure can be driven by temperature. For example, a dissociation fraction and a molecular distortion very similar to those found at 9-fold compression and $3000 \mathrm{~K}$ can be achieved at 6 -fold compression and $10000 \mathrm{~K}$. In addition, we found that lowering the temperature from 3000 to about $300 \mathrm{~K}$ at 9 -fold compression (which we did over a 15 ps long simulation) brings the fluid back to a fully molecular, quasi-ordered solid with a large band gap. (The exact transition temperature to this molecular state has not yet been determined.)

The structural and bonding transformations observed at 9 -fold compression at $3000 \mathrm{~K}$ are accompanied by important changes in the electronic properties of the fluid. Making use of recent algorithmic advances[16], we have computed optical gaps in the liquid using DMC, which in solids[17] and clusters[18, 19] gives optical gaps in excellent agreement with experiment. In Fig. 2 we show the electronic optical gaps averaged over several statistically independent configurations taken from our FPMD simulations, as computed using GGA and DMC at 4-, 6-, and 9-fold compression, at $3000 \mathrm{~K}$. For each atomic configuration we have computed many-body wavefunctions and energies for the ground-state and the lowest excited state using fixed-node DMC[20-22]. Although 
GGA and DMC results are in general rather different in the disordered molecular phase, we find that both DMC and GGA give a gap very close to zero at about 9 -fold compression, within the statistical and finite-size errors of our calculations.

Our results pointing at metallization at 9 fold compression are in agreement with experiments finding metallization of fluid hydrogen at 9-fold compression, at 3000 $\mathrm{K}$. However the calculated pressure is $110 \mathrm{GPa}$, slightly lower than the measured value of $140 \mathrm{GPa}$. This discrepancy could be due to proton quantum effects, not included in our simulations and to experimental uncertainties on the compression ratio at the metallization point. In shock wave experiments pressure is measured but temperature and density are estimated based on empirical models. We note that using a Wigner-Kirkwood $\hbar$ expansion, quantum corrections to the free energy are estimated to be less than $0.5 \%$ at the $\mathrm{P}$ and $\mathrm{T}$ conditions studied here[24].

We also compared total energy differences between insulating and metallic configurations, as obtained within GGA and DMC for selected configurations of our FPMD runs. These differences turned out to be very similar ( $\simeq 0.1 \mathrm{eV} /$ atom $)$, indicating that the GGA description is qualitatively correct and can capture most of the relevant correlation effects present in the ITM region of the fluid.

It is interesting to note that there is a clear correlation (see Table I and Fig. 2) between the values of the electronic gap in the fluid and the fraction of dissociated molecules: the gap decreases and eventually vanishes as more molecules are dissociated. The same correlation was also found at fixed density, e.g. 6-fold compression, where snapshots with the largest (smallest) gap were found to have the biggest (smallest) fraction of dissociated molecules. We also found that as the gap decreases, MLWF spread increases. The role of dissociation in driving gap closure can be further elucidated by simulations where molecules are artificially constrained not to dissociate. In such simulations a gap opening was observed in samples which were metallic in the absence of any constraint on deuteron pairing[25].

As shown in Fig. 2, there is no systematic trend as a function of pressure in the difference between optical gaps computed with DMC and GGA. For example, at six-fold compression, we averaged over snapshots including both mostly molecular and largely atomic-like configurations, with GGA gaps varying from 2 to $0.1 \mathrm{eV}$; no rigid shift between DMC and GGA results obtained for the optical gaps of these configurations was observed. The error of GGA might be related to the localization of the HOMOLUMO states, as noticed in other systems[23].

In order to further compare with experimental data, we computed the DC conductivity of the fluid at $3000 \mathrm{~K}$. This was obtained by taking the zero frequency limit of the frequency dependent conductivity $[\sigma(\omega)]$ computed

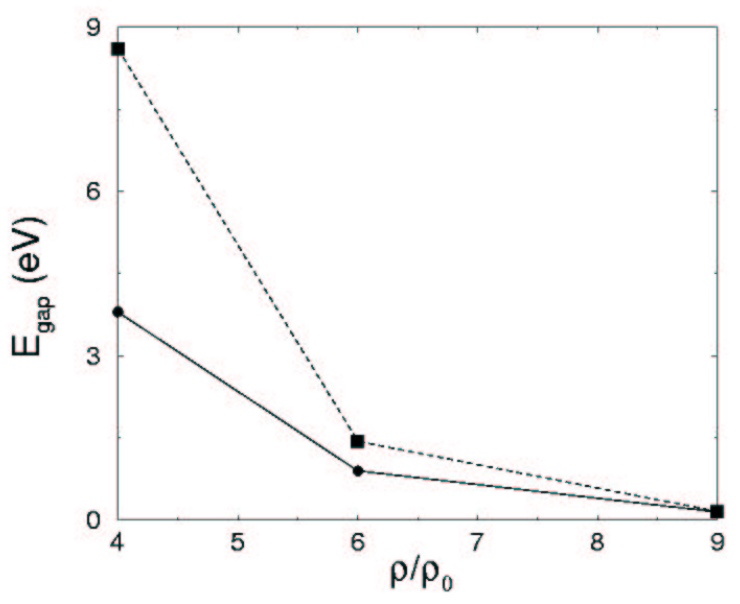

FIG. 2: Electronic energy gaps of liquid deuterium at $3000 \mathrm{~K}$ at 4-, 6-, and 9-fold compression averaged over 7 uncorrelated configurations calculated using the GGA within DFT (circles), and DMC (squares). The lines are a guide to the eye. The DMC statistical error bars are smaller than the symbols.

as a configurational average using the Kubo-Greenwood formula[26]. Figure 3 shows our calculated $\sigma(\omega)$ at 9 -fold compression and $3000 \mathrm{~K}$. Results are shown from simulations using 124 and 992 atoms with a single $\Gamma$ point and from simulations using 124 atoms with $8 \mathrm{k}$ points centered on the $\Gamma$ point. The results with 992 atoms display a Drude-like behavior, unlike the results with only 124 atoms, showing that there are large finite-size effects in the computations of the conductivity. Structural properties (e.g. $g(r))$ and thermodynamic properties such as pressure, on the other hand were found to display much smaller finite-size effects. Extrapolating our results with 992 atoms to zero frequency, we find a DC conductivity of $9300(\Omega \cdot \mathrm{cm})^{-1}$ which is larger than the experimental value of $2000(\Omega \cdot \mathrm{cm})^{-1}$. Previous FPMD simulations[6] with 96 atoms at 9 -fold compression and $3000 \mathrm{~K}$ found $13000(\Omega \cdot \mathrm{cm})^{-1}$ for the DC conductivity, computed with the $\Gamma$ point only. There are several possible sources of the discrepancy between experiment and simulation, some of which have been already mentioned, e.g. experimental uncertainties. In addition we note that use of single particle eigenstates and eigenvalues in the calculations of $\sigma(\omega)$ instead of the many-body wavefunctions and energies introduces an approximation, which is believed to be small but has not yet been quantified.

In summary, we have presented an investigation of the insulator to metal transition in fluid hydrogen based on ab-initio calculations. Using both density functional theory and quantum Monte Carlo, we have shown that the liquid becomes metallic at 9 -fold compression at $3000 \mathrm{~K}$, in agreement with shock wave experiments. The insulator to metal transition is dissociation driven and strongly temperature dependent. When lowering the temperature 


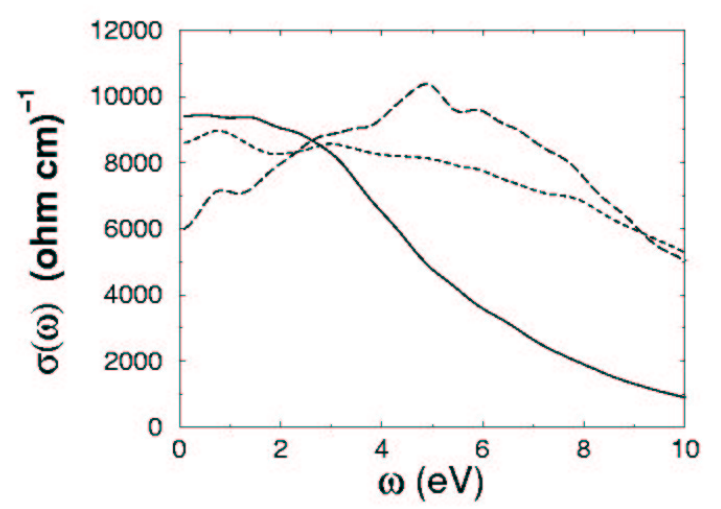

FIG. 3: Frequency dependent electrical conductivity $\sigma(\omega)$ of liquid deuterium at nine-fold compression and $3000 \mathrm{~K}$ averaged over uncorrelated configurations using 992 atoms (solid line) and 124 atoms (dashed line) with $\Gamma$ point sampling of the Brillouin zone; and 124 atoms with $8 \mathrm{k}$ point sampling of the Brillouin zone (dotted line).

at fixed density from 3000 to about $300 \mathrm{~K}$, we found that molecules readily form, and this gives rise to a quasiordered solid. Although not crystalline and not fully ordered, this molecular system is insulating. We therefore conclude that disorder alone cannot induce a ITM in hydrogen at 9-fold compression, as suggested on the basis of simple models, but that the transition is induced by complex structural changes, notably molecular dissociation.

We thank F. Gygi, E.Pollock and A.U.Hazi for useful discussions; B.Militzer, E.Schwegler and P.Loubeyre for a critical reading of the manuscript and J-Y Raty for the conductivity calculations including k-points. This work was performed under the auspices of the U.S. Department of Energy at the University of California/Lawrence Livermore National Laboratory under Contract No. W7405-Eng-48.

[1] K. A. Johnson and N. W. Ashcroft, Nature 403, 632 (2000); B. Militzer and D. M. Ceperley, Phys. Rev. Lett. 85, 1890 (2000); A.Goncharov, E.Gregoryanz, R.Hemley and H-k.Mao, PNAS 98, 14234 (2001) and references therein.

[2] C. Narayana, H. Luo, J. Orloff, and A. L. Ruoff, Nature 393, 46 (1998).

[3] P. Loubeyre, F. Occelli, and R. LeToullec, Nature 416, 613 (2002)

[4] S. T. Weir, A. C. Mitchell, and W. J. Nellis, Phys. Rev. Lett. 76, 1860 (1996).

[5] T. J. Lenosky, J. D. Kress, L. A. Collins, and I. Kwon, Phys. Rev. B 55, R11907 (1997); J. Quant. Spectrosc.
Radiat. Transfer 58, 743 (1997).

[6] O. Pfaffenzeller and D. Hohl, J. Phys.: Condens. Matter 9, 11023 (1997).

[7] L. A. Collins, S. R. Bickham, J. D. Kress, S. Mazevet, T. J. Lenosky, N. J. Troullier, and W. Windl, Phys. Rev. B 63, 184110 (2001); G. Galli, R. Q. Hood, A. U. Hazi, and F. Gygi, Phys. Rev. B 61, 909 (2000).

[8] F. Gygi and G. Galli, Phys. Rev. B 65, 220102 (2002).

[9] M. Ross, Phys. Rev. B 58, 669 (1998).

[10] D. Saumon and G. Chabrier, Phys. Rev. A 46, 2084 (1992).

[11] W. J. Nellis, A. A. Louis, and N. W. Ashcroft, Phil. Trans. R. Soc. Lond. A 356, 119 (1998).

[12] We carried out Car-Parrinello simulations at $T=3000$ and $10000 \mathrm{~K}$ using the JEEP code 1.8.0 (F. Gygi, LLNL 19992002) and deuterium samples. The differences in zeropoint energies between $\mathrm{H}$ and $\mathrm{D}$ are largely smeared out at $3000 \mathrm{~K}$ and $10000 \mathrm{~K}$ and the electronic properties of these two fluids should be identical at the same molar $\rho$ $T$ conditions. The interaction between electrons and ions was described with a norm-conserving pseudopotential [D. Hamann, Phys. Rev. B 40, 2980 (1989)] and only the $\Gamma$ point was used to sample the supercell Brillouin zone (BZ). Single-particle electronic states were expanded in plane waves with a maximum kinetic energy cutoff of 75 Ry. We used a time step of 1-2 a.u. Samples were thermally equilibrated for about 2 ps and then statistics were accumulated for $\simeq 10$ ps.

[13] J. P. Perdew, K. Burke, and M. Ernzerhof, Phys. Rev. Lett. 77, 3865 (1996).

[14] N. Marzari and D. Vanderbilt, Phys. Rev. B 56, 12847 (1997).

[15] I. Souza, R. M. Martin, N. Marzari, X. Zhao, and D. Vanderbilt, Phys. Rev. B 62, 15505 (2000).

[16] A. J. Williamson, R. Q. Hood, and J. C. Grossman, Phys. Rev. Lett. 87, 246406 (2001).

[17] A. J. Williamson, R. Q. Hood, R. J. Needs, and G. Rajagopal, Phys. Rev. B 57, 12140 (1998); M. D. Towler, R. Q. Hood, and R. J. Needs, Phys. Rev. B 62, 2330 (2000).

[18] A. R. Porter, M. D. Towler, R. J. Needs, Phys. Rev. B 64, 035320 (2001).

[19] A. J. Williamson, J. C. Grossman, R. Q. Hood, A. Puzder, and G. Galli, Phys. Rev. Lett. 89, 196803 (2002).

[20] W. M. C. Foulkes, L. Mitas, R. J. Needs, and G. Rajagopal, Rev. Mod. Phys. 73, 33 (2001).

[21] R. J. Needs et al., CASINO version 1.0 User's Manual, University of Cambridge, Cambridge 2000.

[22] A standard Slater-Jastrow form was used for the trial wavefunction. We computed the DMC electronic gap $E^{o p t}=E^{*}-E^{G S}$, where $E^{G S}$ and $E^{*}$ are the total energies of the system in its ground and excited state, and $E^{*}$ is computed by replacing the highest occupied molecular orbital (HOMO) with the lowest unoccupied molecular orbital (LUMO) in the Slater determinant of the trial wavefunction.

[23] A. Puzder, A. J. Williamson, J. C. Grossman and G. Galli, Phys. Rev. Lett. 88, 097401 (2002).

[24] E.Pollock, private communication.

[25] S.Bonev, E.Schwegler and G.Galli (in preparation).

[26] R. Kubo, J. Phys. Soc. Jpn. 12, 570 (1957); D. A. Greenwood, Proc. Phys. Soc. 71, 585 (1958). 